\title{
Microbial Infection Among Admitted Patients of Cardiac Surgery in a Tertiary Hospital Dhaka
}

\author{
M Mustajib Haider ${ }^{1}$ and Hamida Khanum ${ }^{2 *}$ \\ ${ }^{1}$ Cardiac Surgery Department in United Hospital Dhaka, Bangladesh \\ ${ }^{2}$ Parasitology Bramch, Department of Zoology, University of Dhaka, American International University-Bangladesh (AIUB), \\ Bangladesh
}

*Corresponding author: Hamida Khanum, Parasitology Bramch, Department of Zoology, University of Dhaka, American International University-Bangladesh (AIUB), Bangladesh

\section{ARTICLE INFO}

Received: 慧April 23, 2021

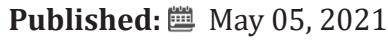

Citation: M Mustajib Haider, Hamida Khanum. Microbial Infection Among Admitted Patients of Cardiac Surgery in a Tertiary Hospital Dhaka. Biomed J Sci \& Tech Res 35(4)-2021. BJSTR. MS.ID.005723.

Keywords: Cardiac Surgery; Microbial Infection; Diabetic; Non-Diabetic and Tertiary Hospital Dhaka

\begin{abstract}
The hospital- acquired infections are among major causes of death and increased morbidity in developed and developing countries resulting to significant burden both for patients as well as public health. Drug resistance is an emerging issue in modern health care. Through this study it was targeted to evaluate, which of the major agents is responsible for infections in the different areas of the body, and which age group and gender are most affected. In 2019, a cross sectional study was carried out among 121 respondents (patients admitted in Cardiac Surgery Department in United Hospital Dhaka, Bangladesh) to assess microbial infections among the admitted patients. The present study was targeted to evaluate, which of the major agents is responsible for infections in the different areas of the body, and which age group and gender are most affected. The study results shows $33.1 \%$ aged $61-70$ years. $50.4 \%$ of normal body weight. Multidrug resistance was found in 3 out of 4 type of organisms. Namely Candida. Klebsiella, Pseudomonas and E coli. The only significant correlation was found between advancement of age with growth of Klebsiella. Microbial infections and their antimicrobial sensitivity profile were assessed by frequency and significance level of $p$ value $<0.05$ was used for statistical analysis (considered as significance), unless specifically mentioned. the $2^{\text {nd }}$ and $3^{\text {rd }}$ generation Cephalosporin was resistant in $100 \%$ of Klebsiella and Pseudomonas positive culture. The only significant correlation was found between advancement of age with growth of Klebsiella.
\end{abstract}

The study found that alarming rate of multidrug resistance present in the organism found in various culture media. This may potentially be useful for future protocol generation and safety measurement applied to patient admitted in cardiac surgery department. All data were analysed using SPSS software versions of 16.0 (SPSS Inc., Chicago, IL, USA.

\section{Introduction}

The hospital- acquired infections are among major causes of death and increased morbidity in developed and developing countries resulting to significant burden both for patients as well as public health. Drug resistance is an emerging issue in modern health care. Especially in case of multi drug resistant organisms. Inherently these bacteria being plasmid and transposon mediated, has facilitated the spread of antibiotic rescinding enzymes to other species of bacteria-. In addition to indiscriminate use of antimicrobials with sub-optimal dose and duration has given rise to more drug resistance [1-3]. Within few years of first isolation of the extended spectrum $\beta$-lactamase producing bacteria, it has spread worldwide and is now found in many different species like Enterobacteriaceae, Pseudomonas aeruginosa, Haemophilus influenzae and Neisseria gonorrhoeae. $\beta$-lactamases producing 
bacteria are increasing in number and causing more severe infections, owing to their continuous mutation [4].

Infectious disease society of America addressed these categories of gram-negative bacilli, namely ESBLS producing E. coli and Klebsiella, MDR Pseudomonas and Carbapenem resistant Acinobactor $\mathrm{sp}$ are priority pathogen causing severity in ICU- [57]. "Without urgent, coordinated action, the world is heading towards a post-antibiotic era, in which common infections and minor injuries, which have been treatable for decades, can once again kill" -reported by WHO (2014) on global surveillance of antimicrobial resistance. The hospital- acquired infections are among major causes of death and increased morbidity in developed and developing countries resulting to significant burden both for patients as well as public health -WHO (2002). The risk of healthcare-associated infection has been estimated to be two to twenty times higher in developing countries than that of resource-rich countries with the percentage of infected patients exceeding 25\% [8-10]. Hospital acquired infection rates in Bangladesh may exceed $30 \%$ in some hospitals [11-13]. Ultimately these resistance pattern results in increasing number of hospitals stay which in turn increases possibilities of Healthcare Associated Infections (HAIs) or hospital-acquired infection. HAIs are often caused by Multi Drug Resistant (MDR) organisms. It is a vicious cycle of suffering for the patient in physical, mental and financial aspect.

In an effort to reduce the development of antibiotic resistance, the recent guidelines by the Infectious Diseases Society of America (IDSA) and the American Thoracic Society (ATS) recommended the regular generation of local antibiogram. According to that recommendations, each hospital should develop and disseminate the antibiogram that is specific to the intensive care population of that hospital. Increasing number of HAIs cases can also be related to the indiscriminate consumption of antimicrobials in primary care in most countries [14] reported that, $30 \%$ to $60 \%$ of antibiotics prescribed in even in ICUs are unnecessary, inappropriate, or suboptimal. Most tertiary care hospital is responsible for treating patients with multiple morbidity and considerably ill individuals. These individuals seeking treatment often requires hospital stay along with various interventions. The infection control unit of hospital has a heaving task to manage the patient's safety and apply different protocols to achieve such goal. Especially in Intensive Care Unit (ICU), where physicians treat serious diseases and debilitated patients. As medical care becomes more complex and antimicrobial resistance increases, cases of HAIs tend to grow [15]. Therefore, a major concern is the increase of microorganisms resistant to a greater number of antimicrobials. They are also associated with significant morbidity, mortality, and increased hospital costs [16]. The slow rate of the antimicrobials invention and development in recent years, highlights the importance of understanding current levels of resistance and preserving the efficacy of existing antibiotics [17].

A cross-sectional study in 1991 on the nosocomial infection rate was $30 \%$ at DMCH [18]. However, in the 2011 report from DMCH that reviewed infection rates on the surgical and burn units identified a $46.2 \%$ rate of nosocomial infections [19]. Wound infections were the most common type of infection followed by upper respiratory tract infection, urinary tract infection, and gastrointestinal infections. Risk factors included older age $(62.5 \%$ of infections), postoperative patients (63.5\% of infections), and an increasing number of visitors per patients per day [20]. The predominant organisms were Acinetobacter spp. (27.6\%), followed by Serratia spp. (17.9\%). The antibiogram showed Acinetobacter spp. were $83 \%$ sensitive only to Colistin followed by Imipenem $67 \%$. High resistances (100\%) were found against Amikacin, Amoxyclavonic acid and Ceftriaxone. Klebsiella pneumoniae were $100 \%$ sensitive to Colistin followed by Imipenem $80 \%$ [21].

However However, the international rise in antimicrobial resistance has weakened the armamentarium. On the other hand, multi drug resistant bacteria, specially multi drug resistant tuberculosis alone cause over 150,000 deaths annually around the world (WHO (2010). Unfortunately, the evolution of drug-resistant microorganisms is unavoidable due to random genetic changes that can render antimicrobials ineffective. While antimicrobial therapy can succeed in killing sensitive pathogens, it also inadvertently selects for organisms that are resistant. Because each exposure to antibiotics contributes to this process, efforts to antibiotic usage restrict only slow the development of resistance [22]. Over the last two decades few new antimicrobials have reached the marketplace. In fact, since 1987 no new class of antimicrobials has been discovered [23]. It is vital to have a microbiological profile for a tertiary care hospital as part of ongoing emergence of these superbugs. The objective of this study was to evaluate, the major agents responsible for infections among admitted patients of Cardiac surgery Department in United Hospital Dhaka Bangladesh, and whether there is any relationship amongst, comorbidity, past history of microbial growth. To observe the microbial infections and their antimicrobial sensitivity profile, 121 patients were included in the study, who were admitted in Cardiac Surgery Department in United Hospital Dhaka based upon a structured questionnaire.

\section{Methods and Materials}

It is a cross-sectional and observational study. The study was carried out in United Hospital Dhaka, Bangladesh. The study was conducted from June 2019 to August 2019 upon patients admitted in Cardiac Surgery Department during the period of June 2019 to August 2019, who had microbiological specimen collected from them during their hospital stay. It was chosen purposively due to 
convenience and feasibility of required study subjects. As this was an observational study specimen were collected microbiological examination from the patients admitted in Cardiac Surgery Department of United Hospital during the period of June 2019 to August 2019. The exploratory analysis of the database included calculation of mean, median, standard deviation and amplitude for independent variables and absolute and relative frequency for dependent variables. IBM SPSS version 17 program was used. Ethical issues: Formal approval was obtained from Ethical Review Board of Amerian International University-Bangladesh (AIUB). Prior to data collection and formal consent was taken from the United Hospital Dhaka, Bangladesh, authority prior to data collection.

\section{Results}

The aim of this study was to evaluate, the major agents responsible for infections in the different areas of the body, and which age group and gender are most affected. Whether there is any relationship amongst blood group, comorbidity, past history of antimicrobial usage with said sensitivity. To observe the microbial infections and their antimicrobial sensitivity profile, 121 patients were included (considering the inclusion criteria) in the study, who were admitted in Cardiac Surgery Department in United Hospital Dhaka based upon a structured questionnaire.

\section{Demographic Information of the Patients}

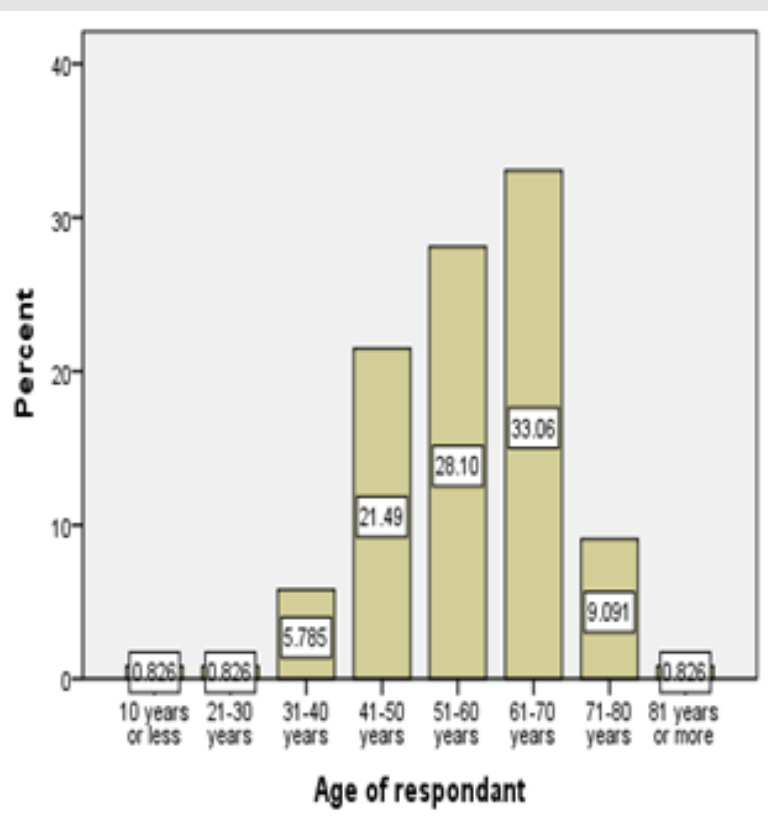

Figure 1: Age group of patients with percent.

Among the study population of $121,84.3 \%$ were male and $15.7 \%$ were female (Table 1). $36.2 \%$ of male between the age 61 to 70 years and $36.8 \%$ females were between 41 to 50 - years of age Figure 1. Age distribution of the patients were $33.1 \%$ between 61-70 years, $28.1 \%$ were between $51-60$ years and $21.5 \%$ between 41-50 years and $0.8 \%$ (Table 1). BMI distributions were $50.4 \%$ were normal body weight, $34.7 \%$ were overweight and 14\% were obese. Interestingly, within the group of patients' ages between 61-70 years, $45 \%$ were both in normal body weight group and in overweight group Figures 2-4. As it was a hospital-based study distribution of gender does not reflect any community hence nonrepresentative. Nevertheless 61-70 years age group male and 41-50 years age group for female were predominant in seeking treatment in Cardiac Surgery Department.

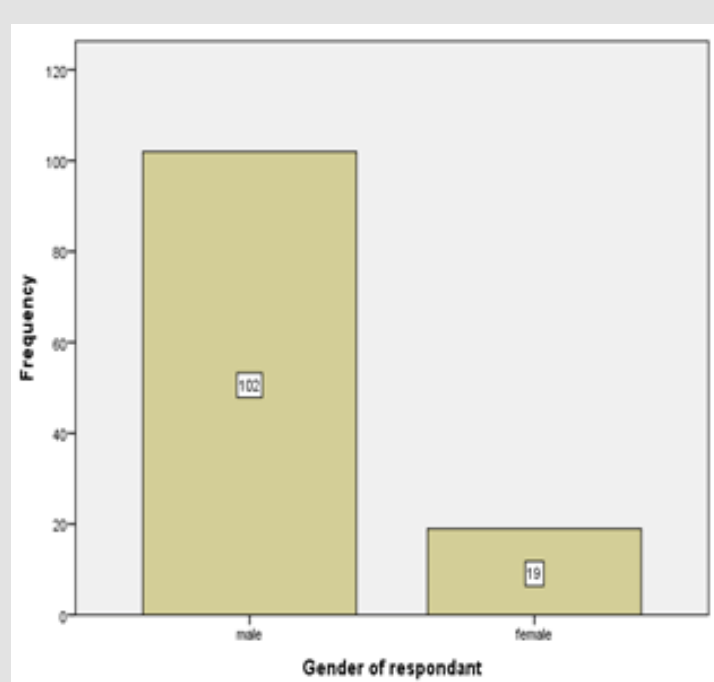

Figure 2: Gender distribution of patients.

Table 1: Descriptive characteristics of the study population, $(\mathrm{N}=121)$.

\begin{tabular}{|c|c|c|}
\hline \multicolumn{2}{|c|}{ Characteristics } & \multirow{2}{*}{$\begin{array}{c}\%) \\
0.8\end{array}$} \\
\hline Age & 10 years or less & \\
\hline & 21-30 years & 0.8 \\
\hline & $31-40$ years & 5.8 \\
\hline & 41-50 years & 21.5 \\
\hline & $51-60$ years & 28.1 \\
\hline & $61-70$ years & 33.1 \\
\hline & $71-80$ years & 9.1 \\
\hline & 81 years or more & 0.8 \\
\hline \multirow[t]{2}{*}{ Gender } & Male & 84.3 \\
\hline & Female & 15.7 \\
\hline \multirow[t]{4}{*}{ BMI } & Underweight & 0.8 \\
\hline & Normal Weight & 50.4 \\
\hline & Overweight & 34.7 \\
\hline & Obese & 14.0 \\
\hline & & \\
\hline
\end{tabular}




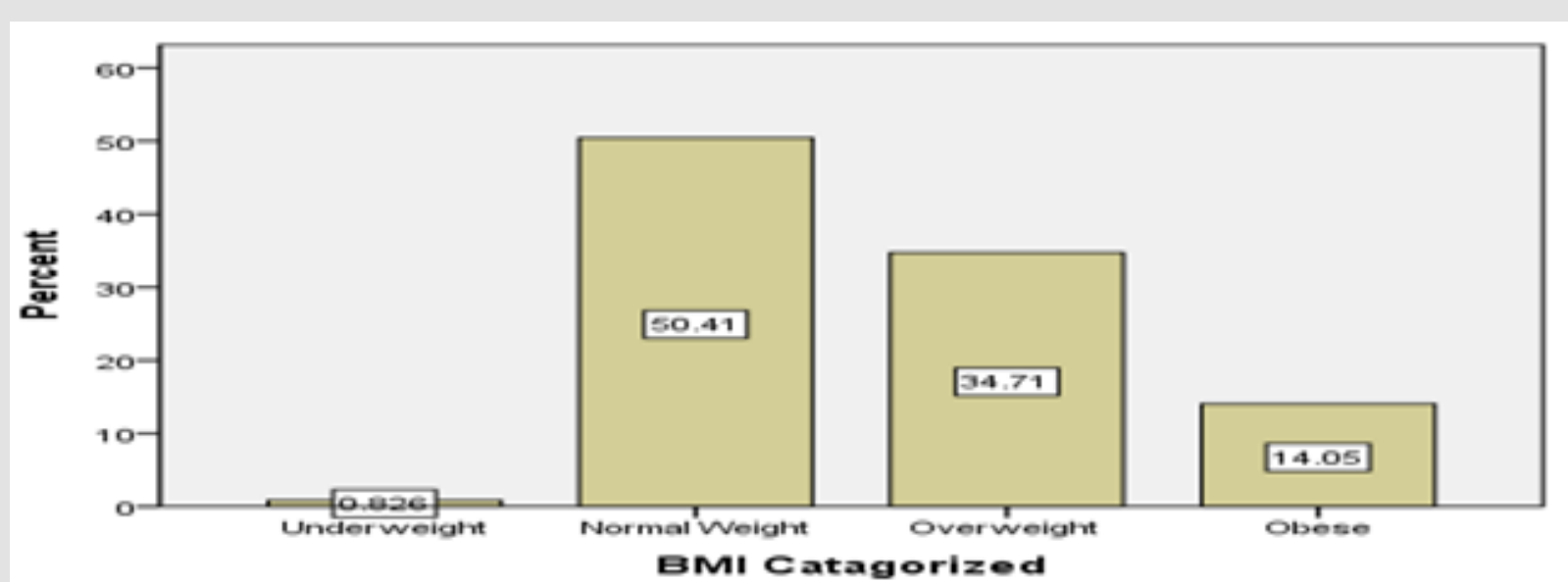

Figure 3: BMI based distribution of respondent patients.

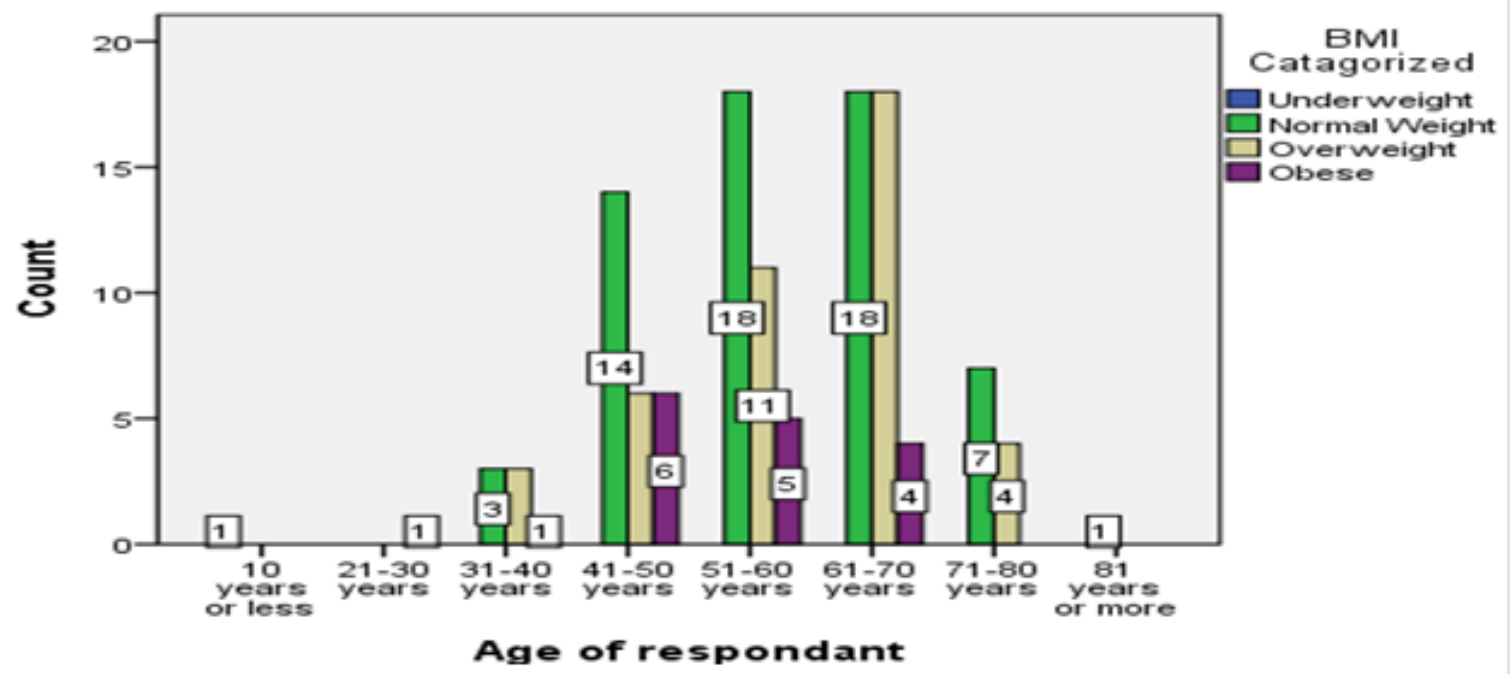

Figure 4: BMI distribution among different age groups of patients.

\section{Microorganisms in Collected Specimen}

Collected specimen revealed mostly (89.3\%) absence of pathogenic microorganisms. However, $10.7 \%$ specimen revealed positive for pathogenic organisms. Only $0.8 \%$ being multiple Organisms in a single case (Table 2). Overall specimen in admitted patients revealed $9.1 \%$ were positive for Candida, $4.1 \%$ positive

Table 2: Microorganism found in different specimen. ( $\mathrm{N}=121)$. for Klebsiella and $\mathbf{1 . 7 \%}$ positive for Pseudomonas (Table 2). Individually these organisms appear in deferent frequency at various specimens. Growth of Candida and Klebsiella was observed only in samples from respiratory tract secretion (Tables 3 \& 4). Pseudomonas found in respiratory secretions and pleural fluid (Table 5). Only one specimen E coli was found in wound sample Figures 6-9.

\begin{tabular}{|c|c|c|c|c|c|c|}
\hline \multirow{2}{*}{ Microorganisms } & \multicolumn{2}{|c|}{ Present } & Organism/ Specimen Absent & \multicolumn{2}{c|}{ Total } \\
\cline { 2 - 7 } & $\mathbf{n}$ & $\mathbf{0}$ & $\mathbf{n}$ & $\mathbf{\%}$ & $\mathbf{N}$ & 121 \\
\hline Candida & 11 & $9.1 \%$ & 110 & $90.9 \%$ & 121 & $100.0 \%$ \\
\hline E coli & 1 & $0.8 \%$ & 120 & $99.2 \%$ & 121 & $100.0 \%$ \\
\hline Klebsiella & 5 & $4.1 \%$ & 116 & $95.9 \%$ & 121 & $100.0 \%$ \\
\hline Pseudomonus & 2 & $1.7 \%$ & 119 & $98.3 \%$ & & $12 \%$ \\
\hline
\end{tabular}


Table 3: Correlations of Age of respondent with Different organisms.

\begin{tabular}{|c|c|c|c|c|c|}
\hline & & Age of Respondent & $\begin{array}{l}\text { Total Candida } \\
\text { Incidence }\end{array}$ & $\begin{array}{l}\text { Total Klebsiella } \\
\text { Incidence }\end{array}$ & $\begin{array}{l}\text { Total Pseudomonus } \\
\text { Incidence }\end{array}$ \\
\hline \multirow{5}{*}{ Age of respondant } & Pearson Correlation & 1 & .105 & $-.024^{*}$ & .092 \\
\hline & Sig. (2-tailed) & & .250 & .794 & .317 \\
\hline & $\begin{array}{l}\text { Sum of Squares and } \\
\text { Cross-products }\end{array}$ & 178.612 & 4.455 & -.702 & 1.719 \\
\hline & Covariance & 1.488 & $.037^{*}$ & $-.006^{* *}$ & $.014^{* *}$ \\
\hline & $\mathrm{N}$ & 121 & 121 & 121 & 121 \\
\hline \multicolumn{6}{|c|}{ **. Correlation is significant at the 0.01 level (2-tailed). } \\
\hline & & rrelation is significa & 0.05 level $(2$-ta & & \\
\hline
\end{tabular}

Table 4: Growth of Klebsiella in different age groups.

\begin{tabular}{|c|c|c|}
\hline Age Group & Characteristics & Culture Positive \\
\hline 10 years or less & Count & 0 \\
\hline 21-30 years & Count & 0 \\
\hline $31-40$ years & Count & 0 \\
\hline \multirow{3}{*}{$41-50$ years } & Count & 2 \\
\hline & $\%$ within Age of respondent & $7.7 \%$ \\
\hline & $\%$ within total Klebsiella incidence & $40.0 \%$ \\
\hline \multirow{3}{*}{$51-60$ years } & Count & 1 \\
\hline & $\%$ within Age of respondent & $2.9 \%$ \\
\hline & \% within total Candida incidence & $20.0 \%$ \\
\hline \multirow{3}{*}{$61-70$ years } & Count & 2 \\
\hline & $\%$ within Age of respondent & $5.0 \%$ \\
\hline & $\%$ within total Klebsiella incidence & $40.0 \%$ \\
\hline 71-80 years & Count & 0 \\
\hline 81 years or more & Count & 0 \\
\hline \multirow[t]{2}{*}{ Total } & Count & 5 \\
\hline & $\%$ within Age of respondent & $4.1 \%$ \\
\hline
\end{tabular}

Table 5: Growth of Pseudomonas in different age groups.

\begin{tabular}{|c|c|c|}
\hline Age group & Characteristics & Percent/ Total \\
\hline 10 years or less & Count & 0 \\
\hline 21-30 years & Count & 0 \\
\hline $31-40$ years & Count & 0 \\
\hline 41-50 years & Count & 0 \\
\hline \multirow{3}{*}{$51-60$ years } & Count & 1 \\
\hline & $\%$ within Age of respondent & $2.9 \%$ \\
\hline & $\%$ within total Pseudomonas incidence & $50.0 \%$ \\
\hline 61-70 years & Count & 0 \\
\hline \multirow{3}{*}{$71-80$ years } & Count & 1 \\
\hline & $\%$ within Age of respondent & $9.1 \%$ \\
\hline & $\%$ within total Pseudomonas incidence & $50.0 \%$ \\
\hline 81 years or more & Count & 0 \\
\hline Total & Count & 2 \\
\hline
\end{tabular}




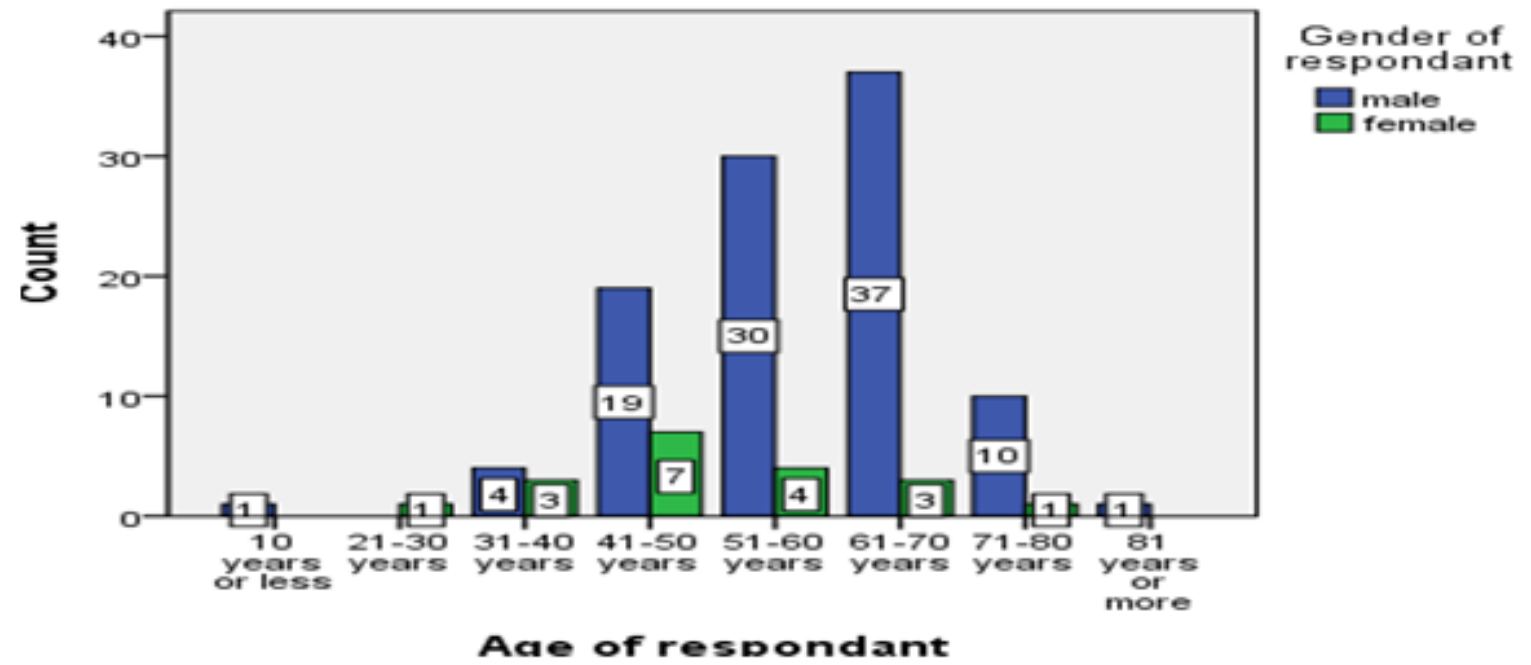

Figure 5: Distribution of Gender among different age groups.

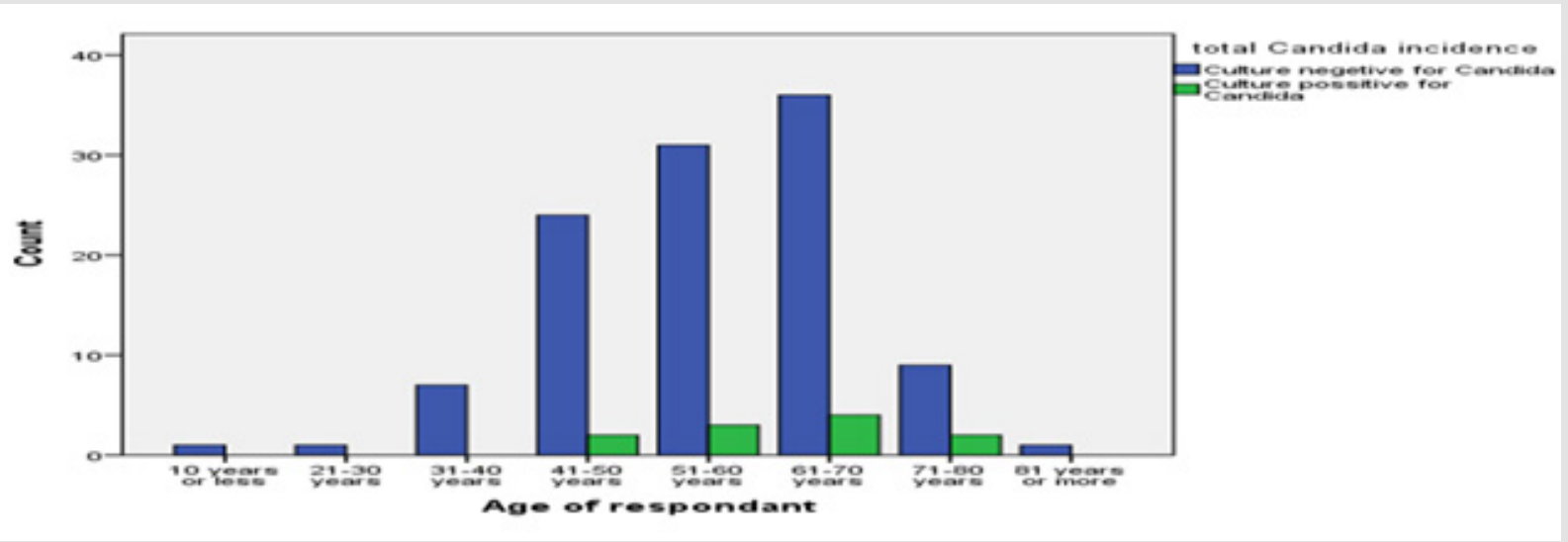

Figure 6: Growth of Candida in different age groups.

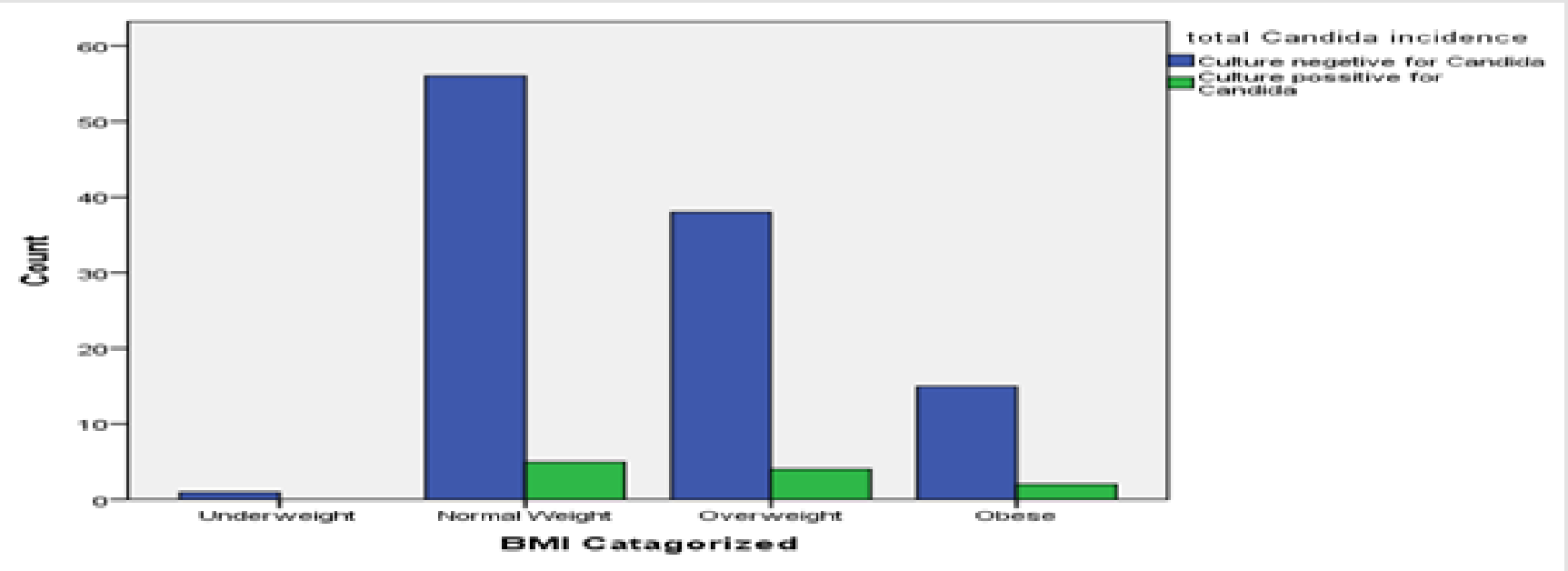

Figure 7: Distribution of Candida in different weight group. 


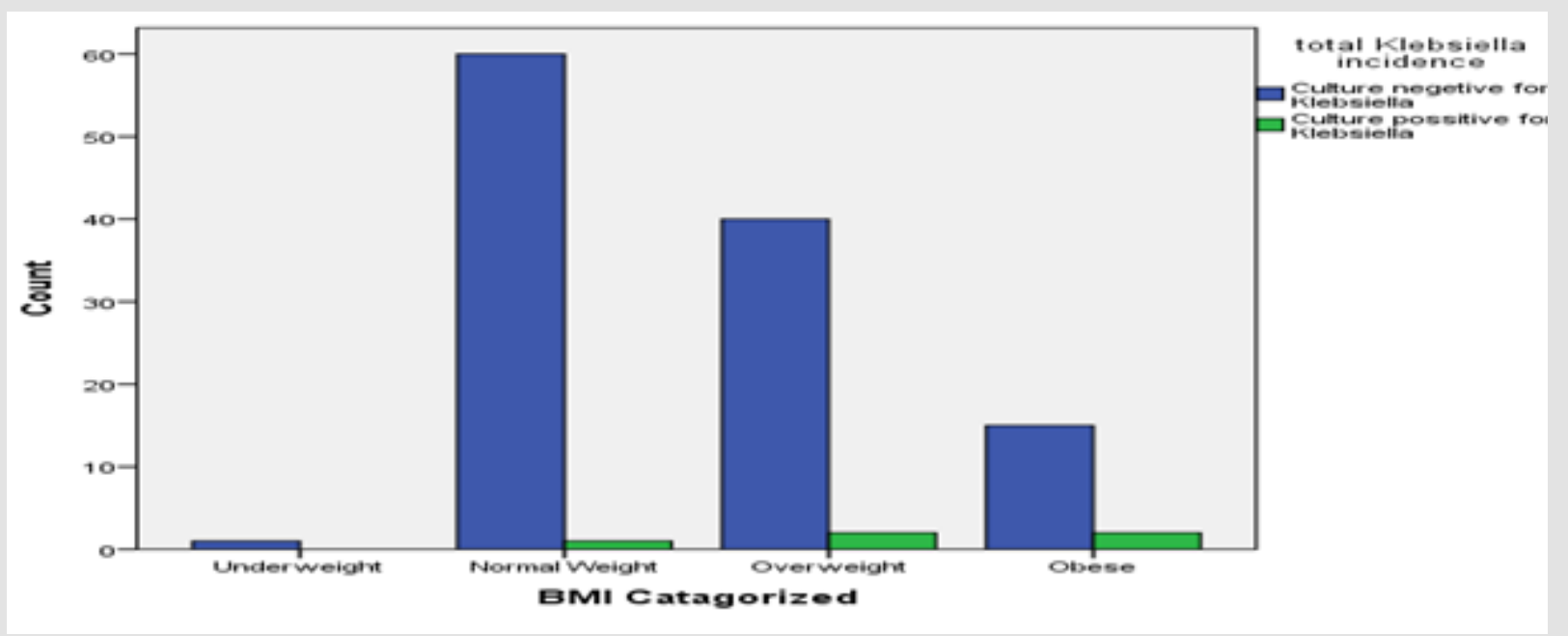

Figure 8: Distribution of Klebsiella in different weight group.

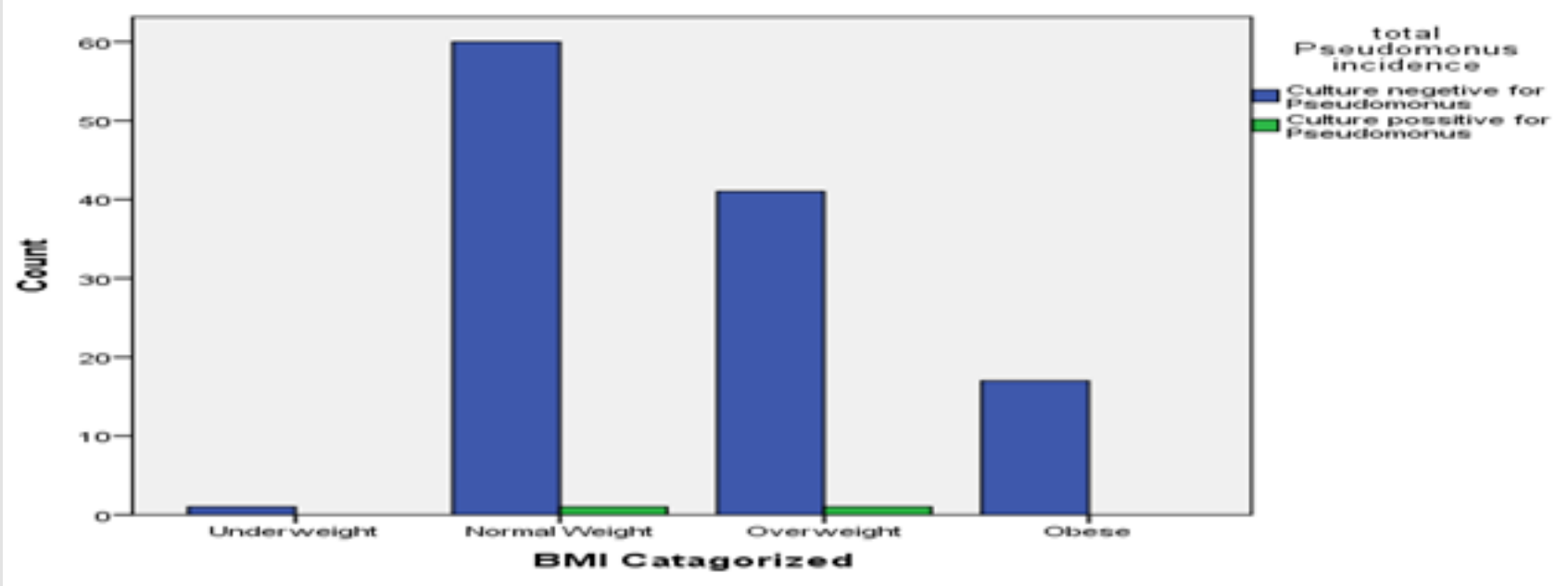

Figure 9: Distribution of Pseudomonas in different weight group.

\section{Microorg`anisms in Different Age Groups}

Candida growth was observed highest among 61-70 years age group (36.4\%), followed by (27.3\%) in 51-60 years group (Table 4 ). Klebsiella was observed at $40 \%$ rate in age group of 41-50 and 6170 years. Pseudomonas was observed equally distributed between age group 51-60 and 71-80 years (Table 5). E coli was observed in a single count in age group 51-60 years. Age was significantly correlated with only Klebsiella growth.

\section{Growth of Microorganisms in Different Weight Groups}

Candida growth was observed to be in $11.8 \%$ in obese groups. Though $45.5 \%$ Candida was found in normal body weight group. It may be considered unremarkable as half of respondents belong in this group. $40 \%$ of Klebsiella was found in in overweight and obese group (Figure 10). Pseudomonas was distributed equally in both normal and over-weight group of respondents. The single case of $\mathrm{E}$ coli was observed in normal body weight group. 


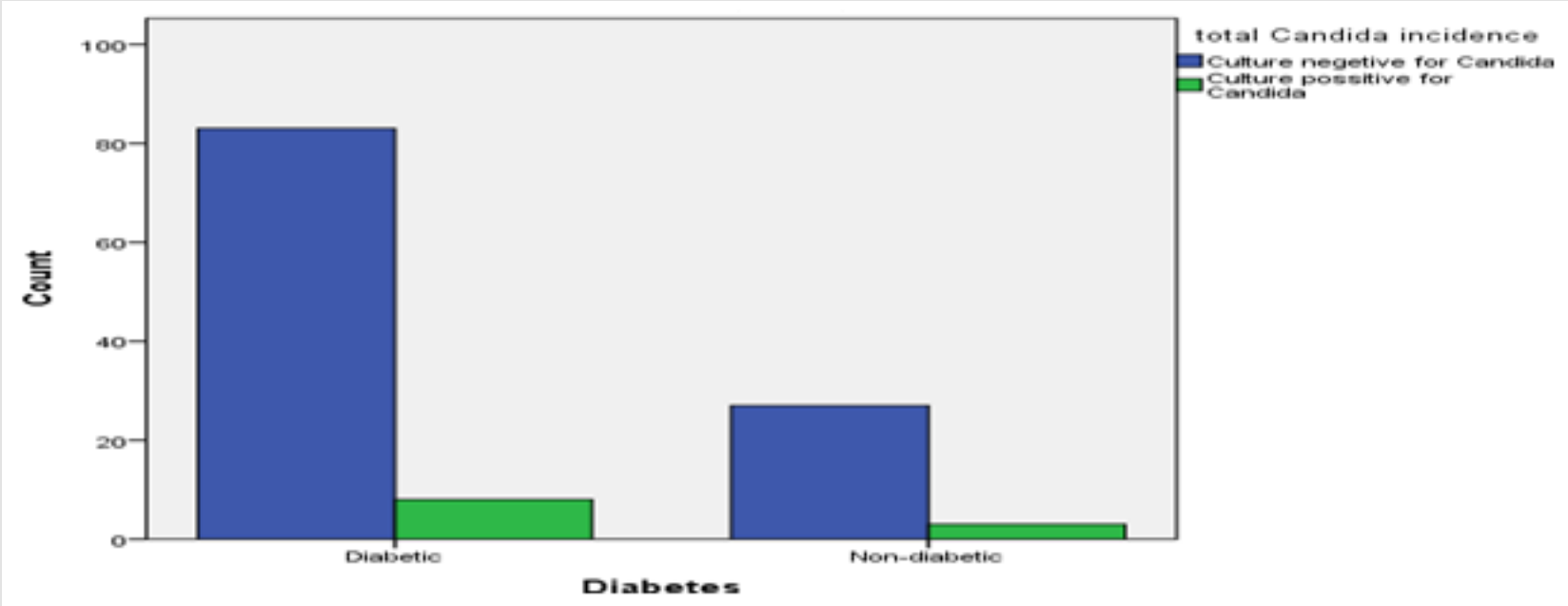

Figure 10: Distribution of Candida in Diabetic and Nondiabetic group.

\section{Discussion}

The purpose of this study was to evaluate, the major agents responsible for infections among admitted patients of Cardiac surgery Department in United Hospital Dhaka Bangladesh [24]. To evaluate microbial growth, multiple questions with different categories were generated and measured through the frequency table and cross tabulation. Demographic data covered age of respondents, gender of the respondents and body weight categories. The survey was administered to patients admitted in Cardiac Surgery Department of United Hospital Dhaka to examine their elements responsible for different microorganism growth from various specimen collected from them. The purpose was also to evaluate the drug sensitivity and resistance to organisms found in culture media [25]. In Bangladesh, Hospital-bed to population ratio is only 0.58:1000 (World bank-(2011) Figure 11-12. Hospital acquired infections take up scarce health care resources by increasing mortality and morbidity, by prolonging patients' hospital stay. Effective infection control strategies can release these resources gone waste, for alternative healthcare uses. In view of the above, this study would be undertaken to address the current situation of antimicrobial resistance and patterns of microorganism responsible for major infection over a period of three months in a four hundred and fifty bed tertiary care hospital of Bangladesh.

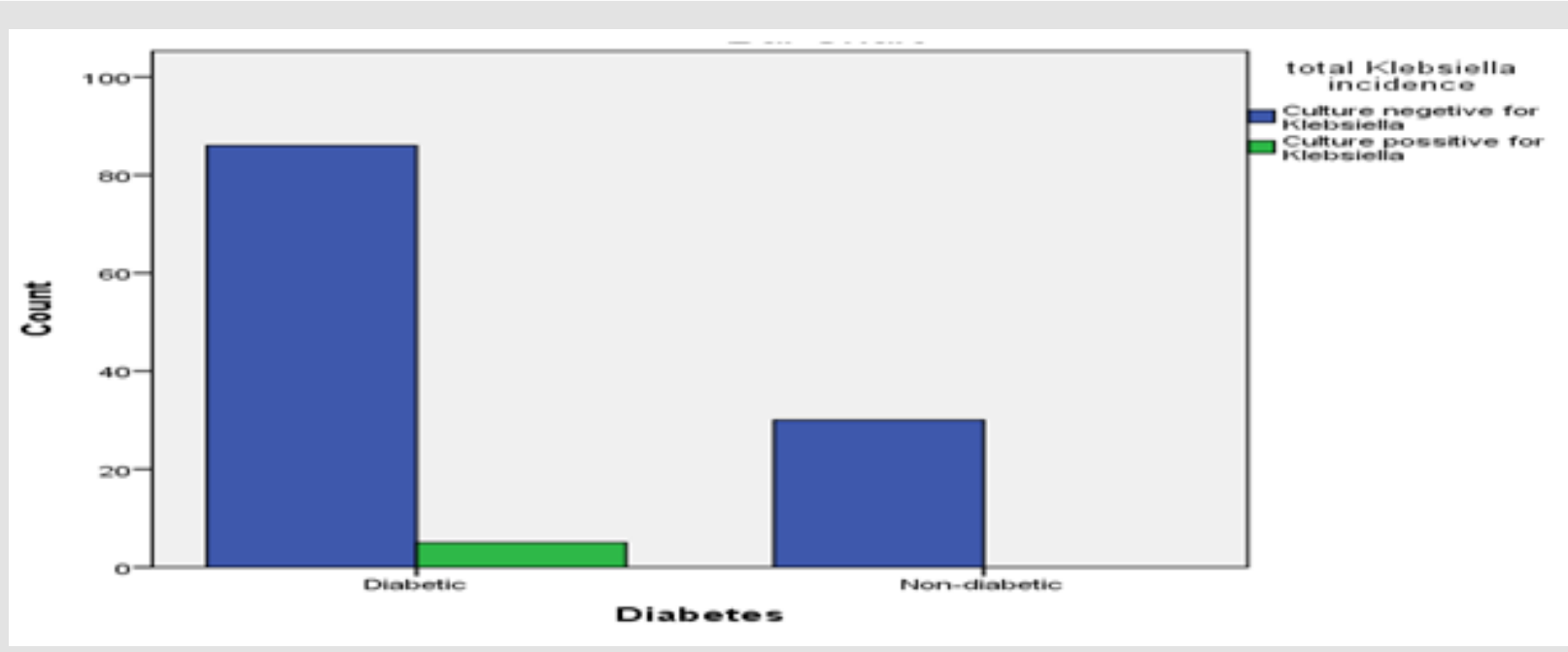

Figure 11: Distribution of Klebsiella in Diabetic and Nondiabetic group. 


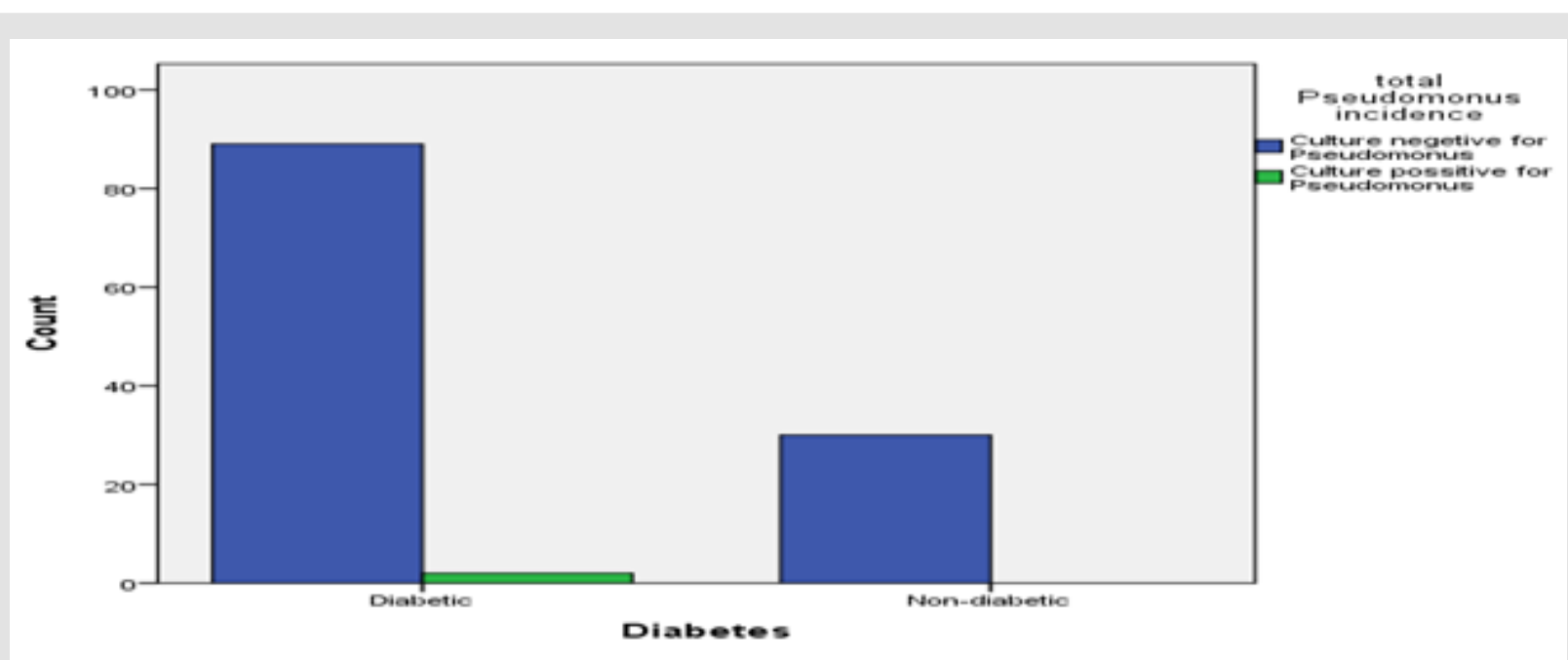

Figure 12: Distribution of Pseudomonas in Diabetic and Nondiabetic group.

In comparison to study conducted in DMCH in $199162.5 \%$ patient with infection were of old age; this study shows all of the microbial growth was present between the age of 41 to more than 81 years of age. The resistance pattern to antibiotics corresponds to this study to at a higher rate. Klebsilla pneumoniae were $100 \%$ sensitive to Colistin followed by Imipenem $80 \%$. Body weight could not be significantly related to any of the organism growth and site of specimen collection. As most of both normal body weight and overweight groups shows equal rate of infections. However, $72.2 \%$ Candida culture positive specimen was collected from Diabetic patients. The study revealed Klebsiella was found to be $100 \%$ sensitive to only Tigecycline. $80 \%$ sensitive to Amikacin, Gentamicin, Meropenem, Netilmicin, Piperacillin. 60\% to Amoxyclave, Azterionam, Levofloxacin and Ciprofloxacin. $40 \%$ to Cefepime, Colistine Sulphate. Only 20\% sensitive to Cotrimoxazole. Pseudomonas growth was analysed and found to be resistant in $100 \%$ capacity with even more antibiotics. Nevertheless, it was found to be $100 \%$ sensitive to Amikacin, Colistine sulphate. $50 \%$ sensitive to Azterionam, Cefepime, Ceftazidime, Levofloxacin, Ciprofloxacin, Gentamicin, Meropenem, Piperacillin and Trecycline. A single case of E coli was also found to be multidrug resistant.

\section{Conclusion}

The patients were evaluated and suggested that $68.6 \%$ patients did not require any specimen collection on clinical ground to suggest infection, $20.7 \%$ of patients who did have clinical indication for culture and sensitivity examination revealed no pathogenic organism to be found, $9.9 \%$ had infection with single culture positive specimen and $0.8 \%$ had multiple organisms present in multiple specimens. This account the hospital acquired infection rate to be $10.7 \%$.
Recommendations:

Some of the recommendations for gap in this research are as follows.

a) In addition to microbial pattern a behavioural study regarding the hygiene practice of the patient and patient attendant could have been observed.

b) Number of attendant and health care personal in contact could be observed for correlation with microbial growth.

c) Severity of IHD should have been accounted for evaluation of the agents responsible for microbial growth.

d) Days of previous hospital stay was not considered as a topic for evaluation, which can provide important correlation between HOIs.

e) Hepatic Insufficiencies along with level of renal impairment and pulmonary function should also be evaluated. As these were not performed as routine investigation for admitted patients and discarded on financial ground.

f) Hospital can put these shortcomings in a protocol to lessen the risk by evaluating those who are of greater risk and take preventative steps.

\section{References}

1. Amin ZA, Nahar N (2017) Hospital Acquired Infection in a Tertiary Military Hospital in Dhaka, Bangladesh. International Journal of Infectious Diseases and Therapy 2(2): 35-39.

2. Bauer A, Kirby WMM, Sherries JC, Turck M (1966) Antibiotic susceptibility testing by standardized single disc method. Am J Clin Pathol 45(4): 493-496.

3. Bonesso MF, Marques SA, Camargo CH, Fortaleza CMCB, Cunha MLRS, et al. (2014) Community-associated methicillin-resistant Staphylococcus aureus in non-outbreak skin infections. Braz J 45(4). 
4. Cavanagh JP, Wolden R, Heise P, Esaiassen E, Klingenberg C, et al. (2016) Antimicrobial susceptibility and body site distribution of community isolates of coagulase-negative staphylococci. APMIS 124(11): 973-978.

5. (2014) Centers for Disease control and Prevention. Core Elements of Hospital Antibiotic Stewardship Programs. 2014.

6. Edelsberg J, Weycker D, Barron R, Xiaoyan Li 3, Hongsheng Wu, et al. (2014) Prevalence of antibiotic resistance in US hospitals. Diagn Microbiol Infect Dis. 78(3): 255-262.

7. Chowdhury AJH, Jahan KR, Sarker MA (2017). wjpls 3(4): 01-06.

8. Faruquzzaman MM (2011) Positive Associations of Nosocomial Infections in Surgical Ward with Etiological Clinical Factors 112(5): 273-237.

9. Grimes DA, Peterson HB, Rosenberg MJ, John I FishburneJr, Roger W Rochat, et al. (1982) Sterilization-Attributable Deaths in Bangladesh. International Journal of Gynecology \& Obstetrics 20(2): 149-154.

10. Hossain MA, Rahman M, Ahmed QS, Malek MA, Sack RB, et al. (1988) Increasing frequency of methicillinam-resistant shigella isolates in urban Dhaka and rural Matlab, Bangladesh: a 6-year observation. Journal of Antimicrobial Chemotherapy 42(1): 99-102.

11. Hussain MA, Fazal MA, Ahmed A (1991) Nosocomial Infection-A Cross Sectional Study in Surgical Wards of Dhaka Medical College Hospital. Journal of Preventive and Social Medicine.

12. Lautenbach E, Synnestvedt M, Weiner MG, Warren B Bilker, Lien Vo, et al. (2009) Epidemiology and impact of imipenem resistance in Acinetobacter baumannii. Infect Control Hosp Epidemiol 30(12): 11861192.

13. Mayon-White RT, Ducel G, Kereseselidze T (1988) An International Survey of the Prevalence of Hospital-Acquired Infection. Journal of Hospital Infection 11(A): 43-48.

14. Perencevich EN, McGregor JC, Shardell M, Jon P Furuno, Anthony D Harris, et al. (2008) Summer peaks in the incidences of Gram-negative

\section{ISSN: 2574-1241}

DOI: 10.26717/BJSTR.2021.35.005723

Hamida Khanum. Biomed J Sci \& Tech Res

This work is licensed under Creative Commons Attribution 4.0 License

Submission Link: https://biomedres.us/submit-manuscript.php bacterial infection among hospitalized patients. Infect Control Hosp Epidemiol 29(12): 1124-1131.

15. Rawat D, Nair D (2010) Extended-spectrum $\beta$-lactamases in Gram Negative Bacteria. J Glob Infect Dis 2(3): 263-274.

16. Sasirekha B, Manasa R, Ramya P, Sneha R (2010) Frequency and antimicrobial sensitivity pattern of extended spectrum ßlactamases producing E. coli and Klebsiella pneumoniae isolated in a tertiary care hospital. Al Ameen J Med Sci 4(1): 22-25.

17. Sepkowitz, Kent A (2011) “One Hundred Years of Salvarsan”. New England Journal of Medicine 365(4): 291-293.

18. Shahida SM, Islam A, Dey BR, Islam F, Venkatesh K, et al. (2016) Hospital Acquired Infections in Low- and Middle-Income Countries: Root Cause Analysis and the Development of Infection Control Practices in Bangladesh 6(1).

19. Tabolt GH (1998) bacterial profile \& antimicrobial susceptibility pattern in catheter related nosocomial infection. Journal of post graduate medicine 44(1): 7-13.

20. Ventola CL (2015) The antibiotic resistance crisis: part 1: causes and threats. P T 40(4): 277-283.

21. (2011) World Bank. Hospital Bed Ratio in Bangladesh. 2011.

22. (2015) World Health Organisation (WHO). Antimicrobial resistance. 2015 .

23. (2010) World Health Organisation (WHO). Global Tuberculosis Control: WHO Report 2010.

24. (2014) World Health Organisation (WHO). Antimicrobial resistance: global report on surveillance. 2014

25. Yusuf MA (2018) Antimicrobial Stewardship: Bangladesh Perspective. Bangladesh J Infect Dis 5(1).

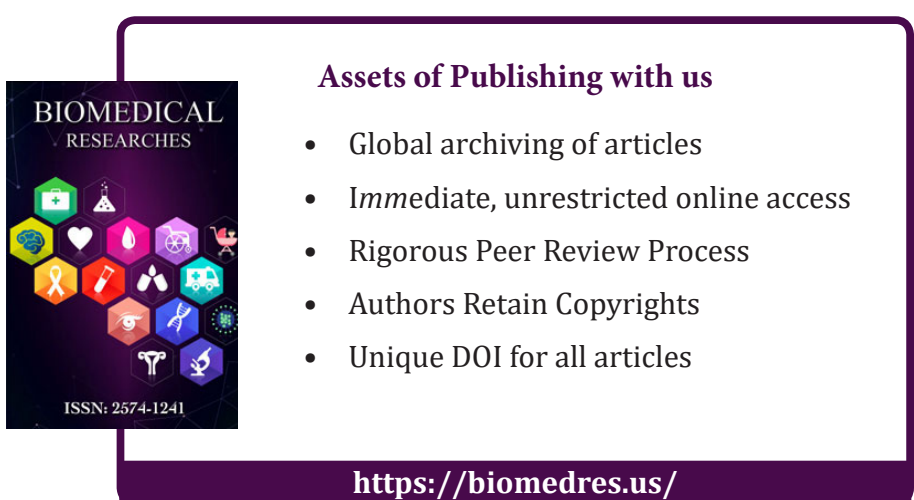

